\title{
Transit timing variability in TrES-1
}

\author{
M. Rabus ${ }^{1}$, R. Alonso ${ }^{2}$, H. J. Deeg ${ }^{1}$, J. A. Belmonte ${ }^{1}$, J. M. \\ Almenara $^{1}$ R. L. Gilliland ${ }^{3}$, and T. M. Brown ${ }^{4}$ \\ ${ }^{1}$ Instituto de Astrofísica de Canarias, \\ Vía Lactea s/n,E-38205 La Laguna - Tenerife, Spain \\ email: mrabus@iac.es \\ ${ }^{2}$ Observatoire Astronomique de Marseille-Provence, \\ 38, rue Frédéric Joliot-Curie,13388 Marseille cedex 13 France \\ ${ }^{3}$ Space Telescope Science Institute, \\ 3700 San Martin Drive, Baltimore, MD 21218, USA \\ ${ }^{4}$ Las Cumbres Global Observatory, \\ 6740 Cortona Dr. Suite 102, Santa Barbara, CA 93117, USA
}

\begin{abstract}
We observed several transits of the exoplanet TrES-1 distributed over four years from 2004 to 2007. On the basis of these observations and additional published data, we present a mid-transit time analysis. The aim is to find indications of the presence of a third body by analysing the difference between the calculated and observed transit times.
\end{abstract}

\section{Introduction}

In 2004, Alonso et al. (2004) discovered the transiting planet TrES-1 around a bright K0V star with an apparent magnitude of 11.79, approximately 157 pc away from us. At that time, TrES-1 was the first planet transiting a bright star that was discovered by a transit-search; and TrES-1 has become one of the most observed and best characterized exoplanet.

Transiting exoplanets offer a possiblity to find additional companions, even down to earth mass, by looking for anomalies in the period (Schneider 2005; Holman \& Murray 2005; Agol et al. 2005). These anomalies are reflected in the times of the transit occurence. Hence, using the ephemerides at a reference epoch and several observations of a transits, it is possible to get the difference 'O-C' between the observed transit time and that calculated with the assumption of constant period. From this difference, we can derive the mass of a possible companion object which is unseen in the transit, but perturbates the period of the transiting planet.

In our current work, we present a study of transit-timing variations (TTV), based on observations and published data. In section 2 we describe the observations and data reduction and the transit time analysis is done in Section 3, followed by the conclusions in Section 4.

\section{Observations and data reduction}

We gathered rapid-cadence, high-precision photometry of TrES-1 transits with the IAC-80 telescope at the Observatorio del Teide, Tenerife, Spain, on eleven nights from 2004 to 2007. All observations were done in the Johnson R filter. We used in the first 
two years the old IAC- 80 CCD and since 2006 the new IAC- 80 CCD with a chip of $2 \mathrm{k} \mathrm{x}$ $2 \mathrm{k}$ and a pixel scale of 0.305 arcsec, resulting in a field-of-view of $10.25 \times 10.25$ arcmin.

The images of each night were bias-subtracted and flat-fielded using standard IRAF procedures. After calibrating the images, we carried out aperture photometry with VAPHOT (Deeg \& Doyle 2001) on the target and several comparison stars of similar brightness within the same CCD frame to obtain the light curves in each night. We also used transit observations with the HST of three visits in Nov. 2004, Jan. 2005 and Mar. 2005.

\section{Mid-transit fitting}

We created different light curve models for IAC-80 R-band and HST observations, respectively. As model we used the FORTRAN routines from Giménez (2006) and the simplex-downhill fitting algorithm Press et al. (1992), minimizing $\chi^{2}$. We adjusted the error in each light curve until we obtained a reduced $\chi^{2}$ of one.

In order to obtain the observed mid-transit time, we derived the best fit of the corresponding model against our data. Our observed mid-transit time was then subtracted from the calculated one, using the ephemerides $2,453,186.8060 \mathrm{HJD}+3.03007^{*} \mathrm{E}$ (Alonso et al. 2004), where $\mathrm{E}$ is the transit number.

The final O-C diagram is shown in Figure 1, where we have excluded O-C values with an error larger than $216 \mathrm{~s}$. We fitted polynomials of different orders to the O-C values, solid, dashed-dotted, and dashed line in Figure 1, respectively. To compare the different polynomial fits, we applied a F-test and found that all polynomial fits describe the O-C values statistically equal well.

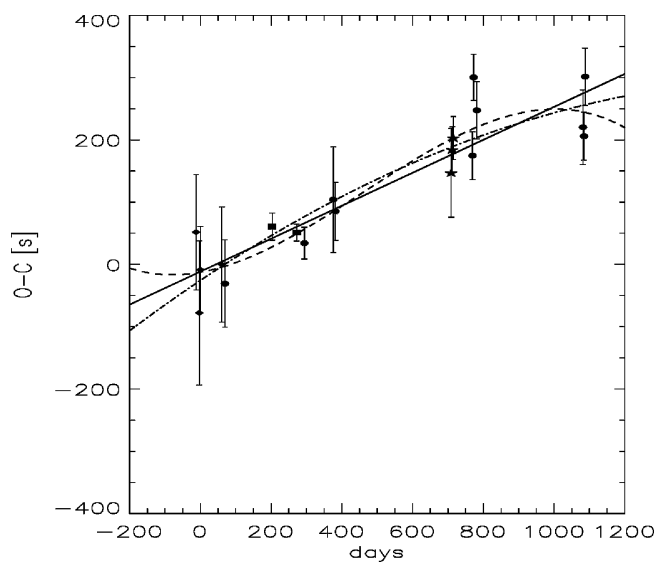

Figure 1. O-C diagram, including O-C values form Alonso et al. (2004) (diamonds), HST observations (squares) and observations from Winn et al. (2007) (stars).

We also used the Scragle algorithm to create a periodogram of the O-C values and to search for transit timing variations between 1 and 300 days, see Figure 2 . In the periodogram we see no clear peak and the maximum measured timing variation is of the order of $85 \mathrm{~s}$. 


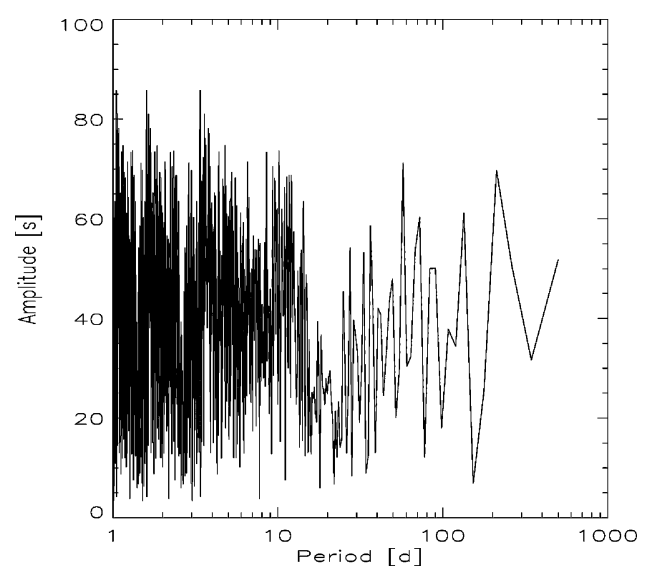

Figure 2. Periodogram based on the Scragle algorithm.

\section{Conclusions}

We checked the period of TrES-1 for variability due to a third non-eclipsing object. There is no clear evidence of a perturbing body for two reasons: all polynomial fits describe the O-C diagram equally well, and the periodogram shows no clear peak. There are two different ways for a perturbing body to cause a timing variation: either the third object can perturb the orbit of the transiting planet, or it can offset the barycenter of the transiting system.

Holman \& Murray (2005) gives an equation to approximate the mass of a body, perturbing the orbit of the transiting planet, for a given timing variation between successive transit:

$$
M_{\text {Pert }}=4.3 \times 10^{-5} \frac{\Delta t}{s}\left(\frac{P_{\text {Pert }}}{d}\right)^{2}\left(1-e_{\text {Pert }}\right)^{3} M_{J},
$$

where $M_{\text {Pert }}$ is the mass in $M_{J}, P_{\text {Pert }}$ the period in days, $e_{\text {Pert }}$ the eccentricity of the perturber and $\Delta t$ is the timing variation in seconds. However, this equation does not consider the huge increase in transit timing of a perturber in mean motion ressonance and is more accurate for eccentricities greater than 0.3 and higher periods of the perturbing body. At mean motion resonance, a smaller-mass body can cause a higher transit timing variation and therefore the mass is overestimated.

For the same system, we can estimate the mass in $M_{J}$ assuming the light time effect with the following equation, see e. g. (Deeg et al. 2000):

$$
M_{\text {Pert }}=92.5 \frac{\frac{\Delta t}{s}}{\left(\frac{P_{P e r t}}{d}\right)^{2 / 3}} M_{J} .
$$

Figure 3 shows an application to the results obtained in this work for TrES-1 for a perturbation of the orbit (solid line) and the time light effect (dashed line). Here we used a timing variation of $85 \mathrm{~s}$ and assumed zero eccentricity. At shorter periods the orbital distortion due to a third body is more dominant, but at a period of 240 days the light time effect becomes more constraining. 


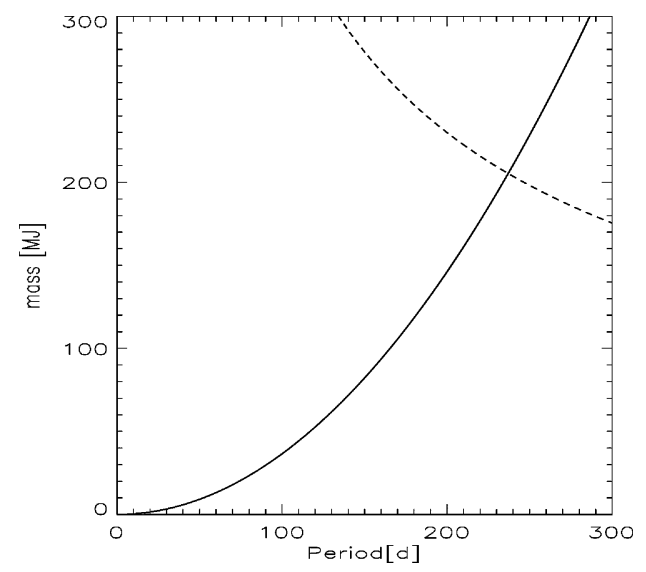

Figure 3. Diagram showing the mass limit in $M_{J}$ for the TrES-1 system applying the measured transit time variation in seconds for different periods in days. Possible masses lie below the curves.

\section{Acknowledgements}

MR like to thank the European Association for Research in Astronomy (EARA) for their support by the EARA - Marie Curie Early Stage Training fellowship.

\section{References}

Agol, E., Steffen, J., Sari, R., \& Clarkson, W. 2005, MNRAS, 359, 567

Alonso, R., Brown, T. M., Torres, G., et al. 2004, ApJ (Letters), 613, L153

Deeg, H. J. \& Doyle, L. R. 2001, Third Workshop on Photometry, ed. W. J. Borucki \& L. E. Lasher, 85

Deeg, H. J., Doyle, L. R., Kozhevnikov, V. P., et al. 2000, A\& A, 358, L5

Giménez, A. 2006, A\&̊A, 450, 1231

Holman, M. J. \& Murray, N. W. 2005, Science, 307, 1288

Press, W. H., et al. 1992, (Cambridge: University Press, —c1992, 2nd ed.)

Schneider, J. 2005, ASP-CS, Vol. 335, ed. C. Sterken, 191

Winn, J. N., Holman, M. J., \& Roussanova, A. 2007, ApJ, 657, 1098 